\title{
Systematic and combined endosonographic staging of lung cancer (SCORE study)
}

\author{
Laurence M.M. Crombag (1) ${ }^{1}$, Christophe Dooms ${ }^{2}$, Jos A. Stigt ${ }^{3}$, \\ Kurt G. Tournoy $y^{4,5}$, Olga C.J. Schuurbiers ${ }^{6}$, Maarten K. Ninaber ${ }^{7}$, \\ Wieneke A. Buikhuisen ${ }^{8}$, Sayed M.S. Hashemi ${ }^{9}$, Peter I. Bonta ${ }^{1}$, \\ Daniël A. Korevaar ${ }^{10}$ and Jouke T. Annema ${ }^{1}$
}

Affiliations: ${ }^{1}$ Dept of Respiratory Medicine, Amsterdam University Medical Center, University of Amsterdam, Amsterdam, The Netherlands. ${ }^{2}$ Dept of Respiratory Disease, University Hospitals KU Leuven, Leuven, Belgium. ${ }^{3}$ Dept of Respiratory Medicine, Isala Hospital, Zwolle, The Netherlands. ${ }^{4}$ Dept of Respiratory Medicine, Onze-Lieve-Vrouw Ziekenhuis, Aalst, Belgium. ${ }^{5}$ Faculty of Medicine and Health Sciences, Ghent University, Ghent, Belgium. 'Dept of Respiratory Medicine, Radboud University Medical Center, Nijmegen, The Netherlands. ${ }^{7}$ Dept of Respiratory Medicine, Leiden University Medical Center, Leiden, The Netherlands. ${ }^{8}$ Dept of Thoracic Oncology, The Netherlands Cancer Institute, Amsterdam, The Netherlands. ${ }^{9}$ Dept of Respiratory Medicine, Amsterdam University Medical Center, Vrije Universiteit Amsterdam, Amsterdam, The Netherlands. ${ }^{10}$ Dept of Clinical Epidemiology, Biostatistics and Bioinformatics, Amsterdam University Medical Center, University of Amsterdam, Amsterdam, The Netherlands.

Correspondence: J.T. Annema, Dept of Respiratory Medicine, G5-247, Amsterdam University Medical Center, University of Amsterdam, P.O. Box 22700, NL-1100 DE Amsterdam, The Netherlands. E-mail: j.t.annemadamc.nl

@ERSpublications

In lung cancer patients, a systematic endobronchial ultrasound (EBUS) combined with an oesophageal investigation using the same EBUS bronchoscope (EUS-B) increases the sensitivity for mediastinal nodal staging by $9 \%$ compared to a targeted EBUS procedure http://ow.ly/aPqF30mJ15q

Cite this article as: Crombag LMM, Dooms C, Stigt JA, et al. Systematic and combined endosonographic staging of lung cancer (SCORE study). Eur Respir J 2019; 53: 1800800 [https://doi.org/10.1183/ 13993003.00800-2018].

ABSTRACT Guidelines recommend endosonography for mediastinal nodal staging in patients with resectable nonsmall cell lung cancer (NSCLC). We hypothesise that a systematic endobronchial ultrasound (EBUS) evaluation combined with an oesophageal investigation using the same EBUS bronchoscope (EUSB) improves mediastinal nodal staging versus the current practice of targeted positron emission tomography (PET)-computed tomography (CT)-guided EBUS staging alone.

A prospective, multicentre, international study (NCT02014324) was conducted in consecutive patients with (suspected) resectable NSCLC. After PET-CT, patients underwent systematic EBUS and EUS-B. Node(s) suspicious on CT, PET, EBUS and/or EUS-B imaging and station 4R, 4L and 7 (short axis $\geqslant 8 \mathrm{~mm}$ ) were sampled. For patients without N2/N3 disease determined on endosonography, surgicalpathological staging was the reference standard.

229 patients were included in this study. The prevalence of N2/N3 disease was 103 out of 229 patients (45\%). A PET-CT-guided targeted approach by EBUS identified 75 patients with N2/N3 disease (sensitivity 73\%, 95\% CI 63-81\%; negative predictive value (NPV) 81\%, 95\% CI 74-87\%). Four additional patients with N2/N3 disease were found by systematic EBUS (sensitivity 77\%, 95\% CI 67-84\%; NPV 84\%, 95\% CI 76-89\%) and five more by EUS-B (84 patients total; sensitivity 82\%, 95\% CI 72-88\%; NPV 87\%, 95\% CI $80-91 \%)$. Additional clinical relevant staging information was obtained in 23 out of 229 patients (10\%).

Systematic EBUS followed by EUS-B increased sensitivity for the detection of N2/N3 disease by $9 \%$ compared to PET-CT-targeted EBUS alone.

This article has supplementary material available from erj.ersjournals.com

This study is registered at ClinicalTrials.gov as NCT02014324. Study data of the SCORE study were de-identified and only used for the present analysis. The data are available for review by request. No predefined data sharing is planned.

Received: April 272018 | Accepted after revision: Nov 072018

Copyright CERS 2019 


\section{Introduction}

Accurate staging of lung cancer is important because it guides treatment and determines prognosis. In the absence of distant metastases, mediastinal nodal status directs treatment [1-3]. Imaging with computed tomography (CT) and (18)F-2-deoxy-2-fluoro-D-glucose (FDG)-positron emission tomography (PET) has limitations in detecting or excluding mediastinal nodal metastases $[4,5]$. Therefore, additional testing with tissue confirmation is often indicated, specifically in case of enlarged or FDG-avid nodes [5-7]. Guidelines recommend endosonography with fine-needle aspiration (FNA) as the initial test for mediastinal nodal tissue staging in nonsmall cell lung cancer (NSCLC). The latest staging guideline endorsed by the European Respiratory Society, European Society of Gastrointestinal Endoscopy and European Society of Thoracic Surgeons advises a combination of endobronchial ultrasound (EBUS) with real-time guided transbronchial needle aspiration (TBNA) and transoesophageal endoscopic ultrasound (EUS)-guided FNA (EUS-(B-)FNA). A complete assessment of mediastinal and hilar nodal stations, including sampling of at least three different mediastinal nodal stations (4R, 4L, 7) for mediastinal nodal staging, is advised [7]. However, there is a lack of strong evidence recommending a combined and systematic staging approach. Current EBUS practice commonly involves the so-called hit-and-run strategy, in which only the (single) node suspected on PET-CT is sampled $[8,9]$. Although quick and straightforward, this approach carries the risk of under-staging. EBUS and EUS investigations are complementary in their diagnostic range, and when combined cover the vast majority of mediastinal and hilar nodes [10]. By combining EBUS-TBNA and EUS-FNA, accuracy for mediastinal metastases increases [11-14]. Importantly, an EUS evaluation can be performed with an EBUS scope (EUS-B), directly following the EBUS procedure and by the same operator, which facilitates a combined approach $[15,16]$.

The objective of the current study was to assess the added value of systematic (assessment of all nodal stations, including routine sampling of stations $4 \mathrm{R}, 7$ and $4 \mathrm{~L}$ ) and combined (endobronchial and oesophageal) endosonographic staging using a single EBUS scope for loco-regional staging (N2/N3 disease), compared to the commonly practiced targeted (PET-CT-guided) EBUS staging alone.

\section{Methods}

\section{Study design and subjects}

The SCORE study is an investigator-initiated, prospective, multicentre diagnostic accuracy study, performed in both university $(n=5)$ and general $(n=3)$ hospitals in the Netherlands and Belgium. Consecutive patients with (suspected) resectable NSCLC who were medically operable were eligible for study inclusion if there was an indication for mediastinal nodal tissue verification according to the current guidelines (mediastinal or hilar nodes with short axis $\geqslant 10 \mathrm{~mm}$, FDG-avid mediastinal or hilar nodes, centrally located lung tumour, or primary tumour and lymph nodes that were not FDG avid) [5-7]. Patients were eligible for study inclusion irrespective of the accessibility of the suspected intrathoracic lymph node to EBUS. Cases with suspected nodes on stations 3A, 5, 6 and 8 were also included. Prior diagnostic evaluation included a conventional workup (medical history, physical examination, laboratory tests and bronchoscopy), CT scan of the chest and integrated PET-CT. Bronchoscopy was performed either prior to endosonography or combined in the same setting with the endosonography procedures. Medical operability was assessed by physical examination, blood tests, pulmonary function tests and/or lung perfusion scans. Surgical resectability and medical operability were verified by specialists from a multidisciplinary team. Patients with proven distant metastasis, irresectable disease (as judged by a thoracic surgeon based on the available imaging) and small peripheral lung tumours without evidence of enlarged or FDG-positive intrathoracic nodes were not considered for eligibility.

Exclusion criteria were mediastinal re-staging after neoadjuvant treatment, active malignancy with a life expectancy of $<2$ years, former therapy for lung cancer (chemotherapy, radiotherapy or surgery), technical contraindication for EBUS or EUS (e.g. oesophagus stenosis), age $<18$ years, pregnancy or inability to consent.

Candidates for study participation were identified at the weekly multidisciplinary lung oncology meeting of the participating centres and provided written informed consent. Prior to endoscopy, the nodal target lesion(s) was defined based on PET-CT imaging. Patients underwent a systematic EBUS followed by an EUS-B procedure [17]. In cases in which nodal mediastinal metastases $(\mathrm{N} 2 / \mathrm{N} 3)$ were found at endosonography, patients were classified as having locally advanced disease (stage III). For patients without pathological evidence of mediastinal metastases at endosonography (N0/N1), surgical verification (mediastinoscopy and/or thoracotomy with nodal dissection) with systematic sampling of mediastinal lymph node stations was performed and considered the reference standard. A multidisciplinary team decided whether to perform surgical staging (mediastinoscopy) or direct surgical lung tumour resection with lymph node dissection. In cases for which no surgical verification was performed (e.g. because the pathology revealed small cell lung cancer (SCLC) or benign disease was suspected), at least 6 months of 
clinical and radiological follow-up including CT scan of the chest was used as an alternative reference standard. The seventh TNM edition of the International Association for the Study of Lung Cancer was used for staging [18].

This investigator-initiated trial was approved by the ethical committees of the eight participating hospitals. This trial was registered as SCORE study (Single scope COmplete staging lung canceR with Endosonography) under NCT02014324 at clinicaltrials.gov.

Single scope combined mediastinal staging procedure: systematic EBUS and EUS-B

EBUS-TBNA and EUS-B-FNA were performed by one operator with a single ultrasound bronchoscope with a linear scanning transducer (Pentax EB-1970UK, Hamburg, Germany; Olympus BF-UC180F, Hamburg, Germany; Fujifilm EB-530US, Düsseldorf, Germany) with patients under conscious, moderate or deep sedation. All endoscopists were trained in both EBUS and EUS-B and had each performed over 200 procedures.

\section{STEP 1: target definition}

Prior to EBUS and EUS-B, the nodal target lesion(s) was defined based on PET-CT imaging, using the report of the radiologist and the PET reader. This was used to report the results of targeted EBUS in the hypothetical scenario in which only the predefined target lesion(s) was sampled.

\section{STEP 2: systematic EBUS}

The endoscopist started with a full, systematic inspection of the mediastinal and hilar lymph nodes located within reach of EBUS using the EBUS assessment tool, identifying (at least) nodal stations $4 \mathrm{~L}, 7,10 / 11 \mathrm{~L}$, $10 / 11 \mathrm{R}$ and $4 \mathrm{R}$ [19]. After inspection, samples were taken from lymph nodes with suspected malignancy (based on ultrasound and/or PET-CT findings, including the defined nodal target lesion) and routine sampling of $4 \mathrm{R}, 4 \mathrm{~L}$ and 7 was performed (if the node short axis was $\geqslant 8 \mathrm{~mm}$ ). Nodal aspirates were taken with $22 \mathrm{G}$ or $25 \mathrm{G}$ needles and from the N3 to N2 to N1 location to avoid upstaging. Suction using a $10 \mathrm{~mL}$ syringe during the aspirations was optional. In the absence of rapid onsite examination, a minimum of two aspirations was performed for each nodal station.

\section{BOX 1 List of definitions}

EBUS-TBNA: Endobronchial ultrasound-guided transbronchial needle aspiration. Investigation of mediastinal and hilar lymph nodes with a linear ultrasound probe from the airways with the possibility of nodal sampling under real-time ultrasound control.

Targeted EBUS-TBNA: Specific investigation for malignancy-suspected mediastinal and/or hilar lymph nodes based ony PET-CT findings (FDG avid or short axis $\geqslant 10 \mathrm{~mm}$ ).

Systematic EBUS-TBNA: Systematic investigation of all mediastinal and hilar lymph nodes according to the endobronchial ultrasound assessment tool, including nodal sampling of suspected nodes (based on PET, CT and/or EBUS) and routine sampling of station $4 \mathrm{R}, 4 \mathrm{~L}$ and 7 (if short axis $\geqslant 8 \mathrm{~mm}$ ).

Endobronchial ultrasound assessment tool (EBUS-AT): Mediastinal and hilar lymph node stations are systematically identified (stations 4L, 7 , 10/11L, 10/11R and 4R).

EUS-B-FNA: Endoscopic ultrasound-guided fine-needle aspiration using the endobronchial ultrasound scope. A systematic investigation of mediastinal lymph nodes with a linear ultrasound probe from the oesophagus according to the endoscopic ultrasound assessment tool, with the possibility of nodal sampling under real-time ultrasound control.

Endoscopic ultrasound assessment tool (EUS-AT): Mediastinal lymph node stations are systematically identified (including stations 4L, 7, 8 and 9). Combined endosonographic staging (EBUS+EUS-B): A systematic EBUS procedure followed by a systematic EUS-B procedure with sampling of lymph nodes suspected on imaging (PET, CT, EBUS and/or EUS-B) and at least stations 4R, $4 \mathrm{~L}$ and 7 (if short axis $\geqslant 8 \mathrm{~mm}$ ). This is a single scope and single operator procedure.

FDG-PET-CT: (18)F-2-deoxy-2-D-glucose-positron emission tomography-computed tomography

Clinically relevant staging information in a lung cancer nodal staging setting is defined as:

- upstaging to a higher N stage, e.g. from N1 to N2, or N0 to N2; or

- increased number of metastatic involved nodal stations, e.g. single-level to multilevel N2 disease.

Suspected lymph node is defined as:

- enlarged on CT (short axis $\geqslant 10 \mathrm{~mm}$ ); or

- FDG avid on PET scan; or

- one or more of the following endobronchial ultrasound/endoscopic ultrasound features: round shape, short axis $\geqslant 10 \mathrm{~mm}$, sharp margins on ultrasound or hypo-echogenic texture.

Sensitivity: The proportion of patients with N2/N3 metastatic nodal spread that is detected by the diagnostic test.

Negative predictive value: The proportion of patients with a negative diagnostic test that do not have N2/N3 metastatic nodal spread.

Negative likelihood ratio: The probability of a patient who has the disease testing negative divided by the probability of a patient who does not have the disease testing negative. 
STEP 3: systematic EUS-B

After the EBUS procedure, the EBUS scope was retracted above the vocal cords and introduced into the oesophagus. Inspection of the mediastinal lymph nodes located within reach of EUS was performed in a systematic way using the EUS assessment tool, identifying the aorta with celiac trunk, the left adrenal gland (if visible) and lymph node stations 7, $4 \mathrm{~L}$ and $4 \mathrm{R}$ (if visible) [20]. Nodal aspirates were taken from suspected lymph nodes and routinely from stations $4 \mathrm{~L}$ and 7 as described above. The nodal stations $4 \mathrm{~L}$ and 7 were sampled from both the endobronchial and oesophageal route if they were suspected on either PET-CT or endosonography.

\section{Cytology of lymph nodes}

Handling of nodal aspirates was performed according to institutional practice. The outcome of the cytological analysis was the presence or absence of malignant cells. The presence of lymphocytes was regarded as proof of a representative lymph node puncture. The presence of malignant cells was considered to be a true positive result because false positive EUS/EBUS findings are extremely rare [21, 22]. Atypical cells in cytology were classified as benign (no malignancy present).

\section{Endpoints}

The primary study endpoint was the sensitivity and negative predictive value (NPV) for detecting mediastinal nodal disease $(\mathrm{N} 2 / \mathrm{N} 3)$ of combined endosonographic staging (by EBUS-TBNA and EUS-B-FNA) in comparison to targeted EBUS-TBNA alone.

The secondary endpoints were 1) the sensitivity, NPV and negative likelihood ratio (NLR) for detecting mediastinal nodal disease $(\mathrm{N} 2 / \mathrm{N} 3)$ of systematic EBUS-TBNA in comparison to PET-CT-directed assessment of the mediastinum (i.e. targeted EBUS); 2) the detection of clinically relevant staging information by combined endosonography (systematic EBUS and EUS-B); 3) the feasibility of detecting the left adrenal gland by EUS-B; 4) serious adverse events of EBUS and EUS-B; and 5) the procedure time for EBUS and EUS-B.

\section{Statistical analysis}

A sample size of 215 was calculated to demonstrate a $7 \%$ increase $(80-87 \%)$ in the sensitivity of detecting loco-regional disease with combined endosonographic staging (systematic EBUS-TBNA+EUS-B-FNA) compared to targeted EBUS staging alone, assuming a prevalence of locally advanced disease (N2/N3) of $50 \%$ and a dropout rate of $5 \%$ with a power of $1-B=0.80$, type 1 error $\alpha=0.05$ and two-sided testing. We calculated estimates of the increase in sensitivity and NPV of targeted EBUS, systematic EBUS and the combined approach. We calculated 95\% confidence intervals around these proportions using the normal approximation. In cases in which an endosonographic procedure could not be performed (e.g. needle aspiration of a specific lymph node could not be performed safely owing to the patient's unrest), the result of that specific procedure was considered to be "negative" (i.e. no mediastinal nodal disease) in the calculation of accuracy estimates of that specific procedure. This was to avoid overestimation of the diagnostic accuracy of the procedure. Patients that did not undergo surgical verification or clinical and radiological follow-up after negative endosonography were excluded from the sensitivity and NPV calculations $(n=4)$. Statistical analyses were performed using IBM SPSS v24.0 (IBM Corp., Armonk, NY, USA).

\section{Results}

Between May 2013 and October 2014, 280 consecutive patients with (suspected) NSCLC were assessed for eligibility. 229 patients (148 men (65\%), mean age 67 years) were included. Baseline characteristics and study flow of enrolled patients are presented in table 1 and figure 1 , respectively.

\section{Final diagnoses}

The prevalence of mediastinal nodal metastases was 103 out of 229 patients (45\%). The final diagnoses of the 229 patients were NSCLC $(n=188 ; 82 \%) ;$ SCLC and large cell neuroendocrine carcinoma $(n=17 ; 7 \%)$; benign, e.g. granulomatous disease, pulmonary infection $(n=14 ; 6 \%)$; suspected lung cancer $(n=8 ; 4 \%)$; and other $(\mathrm{n}=2 ; 1 \%)$ (table 1$)$.

\section{Targeted EBUS (hit-and-run strategy)}

227 patients underwent targeted EBUS with sampling of lymph node(s) suspicious on PET-CT. Mediastinal nodal metastases were detected in 75 patients. In two patients it was not possible to introduce the EBUS scope into the trachea. A median of one hilar and/or mediastinal target lesion(s) was defined prior to endosonography (range 0-8) and a median of one target lesion was sampled (range 0-4). 
TABLE 1 Clinical characteristics of patients in study

\section{Characteristic}

\begin{tabular}{|c|c|}
\hline Patients n & 229 \\
\hline Age mean \pm so years & $67 \pm 8.9$ \\
\hline \multicolumn{2}{|l|}{ Sex } \\
\hline Male & 148 (65) \\
\hline Female & 81 (35) \\
\hline \multicolumn{2}{|c|}{ Diagnostic workup prior to endoscopy } \\
\hline FDG-PET-CT & 211 (92) \\
\hline Bronchoscopy & 135 (59) \\
\hline \multicolumn{2}{|l|}{ Tumour stage PET/CT } \\
\hline cT1 & 73 (32) \\
\hline cT2 & $99(43)$ \\
\hline cT3 & $50(22)$ \\
\hline cT4 & $7(3)$ \\
\hline \multicolumn{2}{|l|}{ Nodal status PET/CT } \\
\hline cNO & 35 (15) \\
\hline $\mathrm{cN} 1$ & $37(16)$ \\
\hline $\mathrm{cN} 2$ & $129(57)$ \\
\hline cN3 & $28(12)$ \\
\hline \multicolumn{2}{|l|}{ Final histopathology data } \\
\hline \multicolumn{2}{|l|}{ NSCLC (epithelial tumours) } \\
\hline Squamous cell carcinoma & 88 (38) \\
\hline Adenocarcinoma & 77 (34) \\
\hline NSCLC-NOS & $21(9)$ \\
\hline Adenosquamous carcinoma & $1(0.5)$ \\
\hline Other: sarcomatoid carcinoma & $1(0.5)$ \\
\hline \multicolumn{2}{|l|}{ Neuroendocrine tumours } \\
\hline Small cell carcinoma & $11(5)$ \\
\hline LCNEC & $6(3)$ \\
\hline Suspected lung cancer ${ }^{\#}$ & $8(3)$ \\
\hline Benign lesion & $14(6)$ \\
\hline Other" & $2(1)$ \\
\hline
\end{tabular}

Data are presented as $\mathrm{n}(\%)$, unless otherwise stated. The 7th TNM edition of the International Association for the Study of Lung Cancer (IASLC) was used for staging. FDG: (18)F-2-deoxy-2-fluoro-D-glucose; PET$\mathrm{CT}$ : positron emission tomography-computed tomography; NSCLC: nonsmall cell lung cancer; NOS: not otherwise specified; LCNEC: large cell neuroendocrine carcinoma. ${ }^{\#}$ : suspicion of lung cancer based on clinical presentation in combination with imaging characteristics (PET-CT) in the absence of tissue proof of malignancy; ": lymphoma and metastasis of extrathoracic tumour.

\section{Added value of systematic EBUS}

215 patients underwent a systematic EBUS procedure with systematic inspection of the mediastinal and hilar lymph nodes, revealing four additional patients with mediastinal disease compared to PET-CT-guided targeted EBUS (three on station 7, and one on both station 7 and 4R). In 14 patients, systematic EBUS (including routine sampling) could not be performed, owing to cough or anxiety of the patient. Systematic EBUS revealed clinically relevant staging information in four more patients through the detection of multilevel N2 disease (instead of single-level N2 disease) in three patients and upstaging to $\mathrm{N} 3$ disease in one patient (figure 2 and supplement 1). A median of two different mediastinal nodal stations (range 0-4) were sampled. A median of three (range 1-9) lymph node passes per nodal station were taken. The median EBUS procedure time was 20 min (range 0-68).

\section{Added value of systematic EUS-B (following systematic EBUS)}

220 patients underwent a systematic EUS-B procedure after the endobronchial procedure, revealing pathological proof of mediastinal metastases in five patients not found by systematic EBUS. These were located in stations $4 \mathrm{~L}(\mathrm{n}=2)$, subcarinal station $7(\mathrm{n}=2)$ and station $8(\mathrm{n}=2)$ (in one patient additional nodal metastases were found in two different nodal stations). In nine patients no systematic oesophageal procedure was performed either because the endoscopist was unable to introduce the EBUS scope into the oesophagus, or the EUS-B procedure was aborted because of patient unrest. EUS-B also revealed clinically relevant staging information in 11 more patients through the detection of multilevel N2/N3 disease (instead of single-level N2/N3 disease) in nine patients and upstaging to N3 disease in two patients (figure 2 and supplement 2). At EUS-B a median of one different mediastinal nodal station (range 0-3) was 


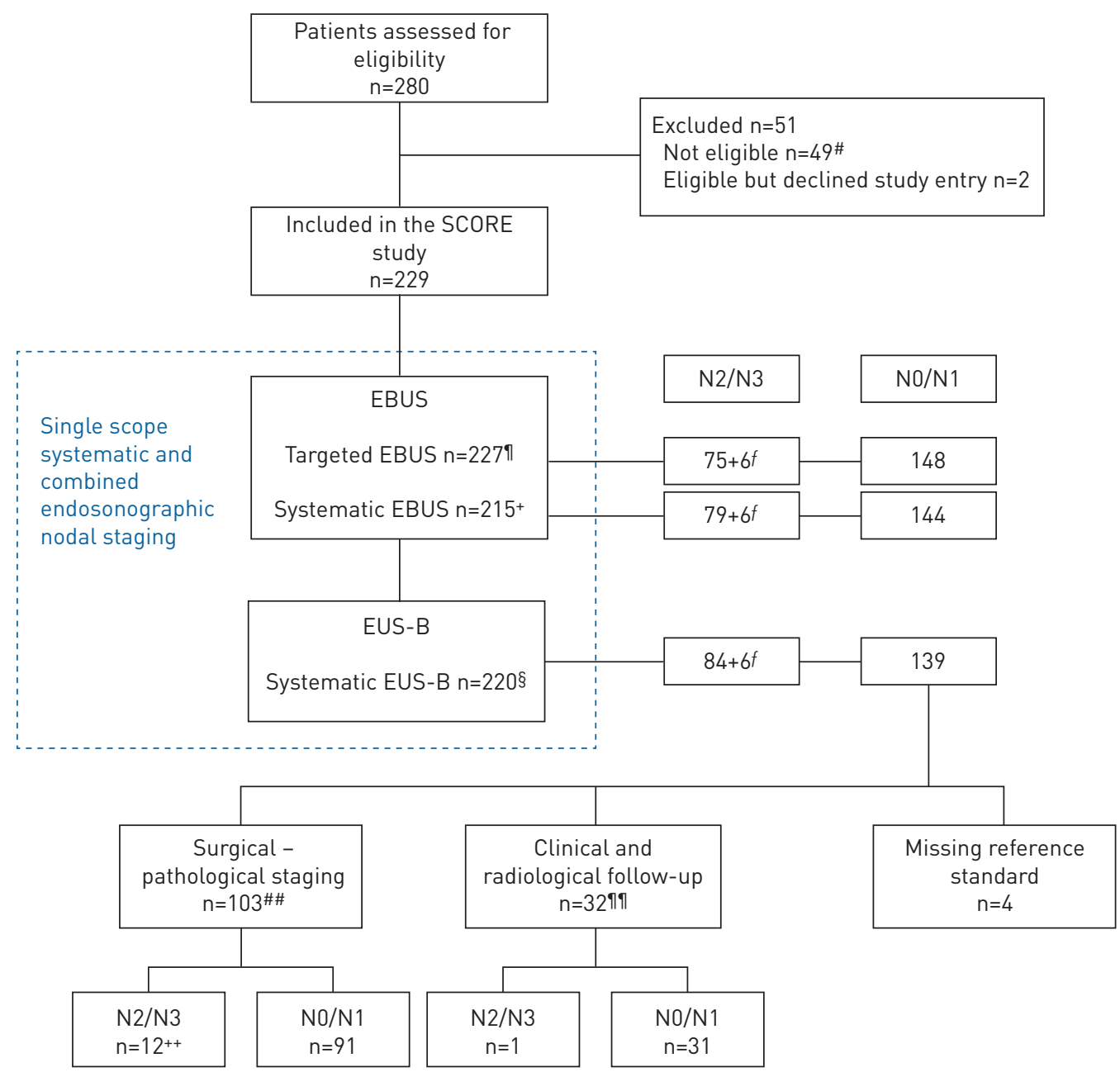

FIGURE 1 Enrolment of patients with (suspected) lung cancer and an indication for mediastinal nodal tissue staging who underwent endobronchial ultrasound (EBUS) followed by endoscopic ultrasound using the EBUS scope (EUS-B). \#: of 49 patients ineligible for study participation, 32 were deemed as medically inoperable, eight had previous therapy for lung cancer and nine had an indication for induction therapy; ${ }^{\text {? }}$ : in two out of 229 patients (1\%), the endoscopist was not able to introduce the EBUS scope into the trachea; ${ }^{+}$: systematic EBUS was not possible in 14 patients $(6 \%)$ owing to cough and/or unrest, including the two patients in whom the introduction of the EBUS scope into the trachea failed; ${ }^{\S}$ : systematic EUS-B was not possible in nine patients (4\%) because the EBUS scope could not be introduced into the oesophagus or because of patient unrest; ${ }^{f}:$ in six additional patients, a diagnosis of N2/N3 (all in stations 5 and 6 ) was made based on PET-CT and endosonography characteristics (round shape, enlarged, sharp margins and hypo-echogenic), because the suspected mediastinal lymph node could only be reached by a transvascular approach (station 5 and 6) (the results of these six patients were considered false negative for sensitivity and NPV calculations); \#\#: delayed surgery in three patients after induction therapy; १ी: $^{\text {\# }}$ treating physician or multidisciplinary team refrained from surgery with lymph node dissection because of nonmalignant disease $(n=7$, diagnosis based on wedge resection, punctures, cultures and/or significant reduction on follow-up imaging), tumour board decision (medically inoperability $n=8$; irresectable tumour $n=2$; radiation \pm chemotherapy $n=10$, e.g. for small cell lung cancer), patient refusal ( $n=5)$, deceased before surgery (cerebrovascular accident, $n=1$ ); pleuritis carcinomatosa at surgery, no lymph node dissection performed $(n=1)$; perioperative $T 4$, no lymph node dissection performed $(n=1)$; pulmonary metastasis of extrathoracic tumour $(n=1) ;{ }^{++}$: one patient underwent surgical staging after induction chemotherapy revealing no mediastinal metastases, but lymph node decreased in size after induction therapy and therefore endosonography was considered to be false negative; thus, endosonography results were false negative in 12 patients.

sampled. A median of two (range 1-6) lymph node passes per nodal station were taken. The median procedure time was $10 \mathrm{~min}$ (range 0-45). A mean of 1.4 needles was used (range 0-3) for the combined EBUS and EUS-B procedure. Rapid onsite examination was available in one third of patients.

Reference standard and false negative endosonographic findings

In 84 patients EBUS and/or EUS-B revealed pathological proof of N2 disease (true positive). In six other patients a diagnosis of N2/N3 (all in station 5/6) was made based on the combination of PET-CT 


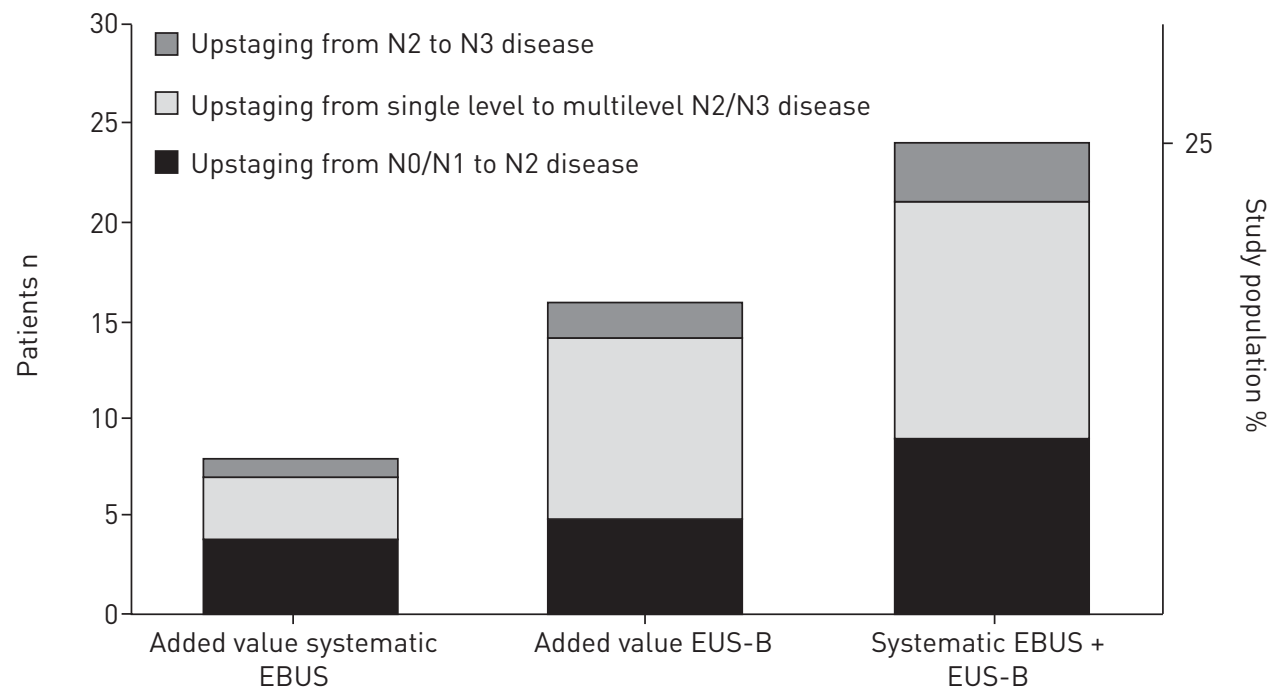

FIGURE 2 Additional clinical staging information of systematic and combined endosonography compared to targeted endobronchial ultrasound (EBUS) $(n=229)$. The left bar reflects the number of patients in whom additional clinical staging information was found by performing a systematic EBUS procedure after a targeted EBUS procedure (only sampling the nodes suspected on imaging). The middle bar shows the number of patients in whom additional clinical staging information was found by performing an endoscopic ultrasound using the EBUS scope (EUS-B) procedure after a systematic EBUS procedure. The right bar shows the number of patients in whom additional clinical staging information was found by performing a combined systematic EBUS and EUS-B procedure compared to a targeted EBUS. Clinical relevant staging information was defined as upstaging to a higher $\mathrm{N}$ stage, e.g. from $\mathrm{N} 1$ to $\mathrm{N} 2$ or $\mathrm{N} 2$ to $\mathrm{N} 3$, and/or increased number of metastatic involved nodal stations, e.g. single-level to multilevel N2 disease. Systematic EBUS and EUS-B both provided added value for one patient.

and endosonography characteristics (round shape, enlarged, sharp margins and hypo-echogenic). Owing to position of these lymph node stations, adequate and safe sampling was not possible because of the interposition of vascular structures. To calculate sensitivity and NPV, the results of these six patients were considered false negative in order to avoid overestimating the accuracy of the endosonography procedures.

Of the 139 patients in whom endosonography revealed no mediastinal metastases (N0/N1), 103 (74\%) underwent surgical-pathological verification and 32 (23\%) underwent clinical and radiological follow-up. In four patients (3\%) a reference standard for mediastinal metastasis was missing (due to death in three) (figure 1). Surgical nodal verification was performed by mediastinoscopy, video-assisted thoracoscopic surgery or thoracotomy with lymph node dissection. At surgical verification, a median of four different hilar and mediastinal nodal stations (range 0-8) was sampled, revealing nodal metastases in 11 patients. One patient underwent surgical staging after induction chemotherapy, revealing no mediastinal metastases, but after induction therapy the lymph node decreased in size and therefore endosonography was considered to be false negative. In one patient radiological follow-up of mediastinal lymph nodes showed growth of one mediastinal lymph node suspicious for nodal metastases after chemoradiotherapy. The endosonography result of this patient was assumed to be false negative. The median duration of radiological and clinical follow-up was 8 months (range 3-24 months). At preoperative staging, combined EBUS and EUS-B results were false negative for $\mathrm{N} 2 / \mathrm{N} 3$ disease in 13 patients (13\%). In five out of 13 patients $(38 \%)$ these nodes were located in stations 5, 6 and $3 \mathrm{~A}$, beyond the reach of endosonography, and in three cases only concerned micro metastases (supplement 3 ).

\section{Outcomes}

The sensitivity for detecting mediastinal nodal metastases by PET-CT-guided targeted EBUS staging alone was $73 \%$ (75 out of 103 ; 95\% CI 63-81\%), for systematic EBUS was 77\% (79 out of 103 ; 95\% CI $67-84 \%$ ) and for combined endosonography (systematic EBUS+EUS-B) was 82\% (84 out of 103; 95\% CI 72-88\%). The NPVs of targeted EBUS, systematic EBUS and combined endosonography (systematic EBUS+EUS-B) were $81 \%$ (122 out of $150 ; 95 \%$ CI $74-87 \%$ ), $84 \%$ (122 out of $146 ; 95 \%$ CI $76-89 \%$ ) and $87 \%$ (122 out of 141; 95\% CI 80-91\%), respectively. The NLRs of targeted EBUS, systematic EBUS and combined endosonography (systematic EBUS+EUS-B) were 0.27 (95\% CI $0.20-0.37$ ), 0.23 (95\% CI $0.16-0.33$ ) and 0.18 (95\% CI 0.12-0.28), respectively (table 2). 


\begin{tabular}{|c|c|c|c|c|c|}
\hline \multirow[t]{2}{*}{ Nodal invasion, N2/N3 disease } & \multicolumn{2}{|c|}{ Sensitivity } & \multicolumn{2}{|c|}{$\begin{array}{c}\text { Negative predictive } \\
\text { value }\end{array}$} & \multirow[t]{2}{*}{$\begin{array}{l}\text { Negative likelihood } \\
\text { ratio }(95 \% \mathrm{CI})\end{array}$} \\
\hline & $\mathrm{n} / \mathrm{N}$ & $\%(95 \% \mathrm{Cl})$ & $n / N$ & $\%(95 \% \mathrm{Cl})$ & \\
\hline Targeted EBUS & $75 / 103$ & $73(63-81)$ & $122 / 150$ & $81(74-87)$ & $0.27(0.20-0.37)$ \\
\hline Systematic EBUS & $79 / 103$ & 77 (67-84) & $122 / 146$ & $84(76-89)$ & $0.23(0.16-0.33)$ \\
\hline Combined EBUS+EUS-B & $84 / 103$ & $82(72-88)$ & $122 / 141$ & $87(80-91)$ & $0.18(0.12-0.28)$ \\
\hline
\end{tabular}

In six patients, mediastinal nodal spread was based on positron emission tomography-computed tomography and endosonographic characteristics only, without pathological proof. For calculating sensitivity, negative predictive value and negative likelihood ratio, the endosonography results of these six patients were considered false negative. Considering these specific six patients as true positives would result in a sensitivity of targeted EBUS versus systematic EBUS versus combined EBUS+EUS-B of $79 \%$ versus $83 \%$ versus $87 \%$ and negative predictive values of $85 \%$ versus $87 \%$ versus $90 \%$. Patients that did not undergo surgical verification nor clinical and radiological follow-up after negative endosonography were excluded from calculations of sensitivity, negative predictive value and negative likelihood ratio. EBUS: endobronchial ultrasound; EUS-B: endoscopic ultrasound using the EBUS scope. \#: combined EBUS and/or EUS-B detected N2/N3 disease in 90 patients out of 225 .

Systematic EBUS revealed clinically relevant staging information in eight patients compared to targeted EBUS. In 16 patients, EUS-B added clinically relevant staging information to the systematic EBUS procedure (figure 2). There was added value for one patient for both systematic EBUS and EUS-B (supplements 1 and 2). 10 patients needed to undergo a systematic EBUS followed by a EUS-B procedure to detect clinically relevant mediastinal nodal staging information compared to the hit-and-run targeted EBUS procedure.

In 94 out of 151 patients (62\%) in whom visualisation the left adrenal gland was attempted, it was possible to detect the left adrenal gland by EUS-B.

No serious adverse events related to the endosonography procedures were reported. One patient had a fatal intracranial bleeding $48 \mathrm{~h}$ after endosonography. This was judged as unlikely to be related to the endosonography procedure.

\section{Discussion}

A systematic EBUS procedure followed by EUS-B increases the sensitivity for detecting mediastinal nodal metastases in lung cancer patients by $9 \%$ compared to a PET-CT-guided targeted EBUS approach. Furthermore, clinically relevant staging information is obtained in one out of 10 patients. EUS-B prolongs the endosonography procedure by $10 \mathrm{~min}$ and the benefit in staging was not associated with complications.

We showed that a targeted EBUS procedure based on PET-CT findings has a sensitivity for detecting mediastinal nodal disease $(\mathrm{N} 2 / \mathrm{N} 3)$ of $73 \%$. This is comparable with a large meta-analysis in which a mean sensitivity of $72 \%$ (95\% CI 58-82\%) for EBUS was reported [23]. By performing a systematic EBUS, sensitivity increased by $4 \%$ in comparison to a targeted EBUS approach. To the best of our knowledge, systematic and targeted mediastinal staging with EBUS-TBNA have previously not been directly compared in a prospective study. A retrospective study in 93 patients reported on systematic nodal sampling in EBUS and concluded that routinely sampling more than two mediastinal stations may improve staging [24]. A recent single-centre retrospective study reported a $13 \%$ increase in important clinical information by systematic EBUS sampling compared to targeted EBUS [25]. We also found that systematic evaluation including routine sampling of (at least) stations $4 \mathrm{R}, 7$ and $4 \mathrm{~L}$, if the short axis $\geqslant 8 \mathrm{~mm}$, increased sensitivity for nodal staging compared to PET-CT-guided targeted EBUS alone. By introducing the EBUS scope into the oesophagus following a systematic EBUS procedure, sensitivity for detecting mediastinal metastases increased by another 5\%. A recent systematic review showed that the addition of EUS(-B) to EBUS leads to a $12 \%$ gain in the detection of mediastinal nodal metastases [23]. A possible explanation for the lower difference found in the SCORE study is the fact that systematic EBUS with routine sampling of mediastinal lymph nodes was performed prior to EUS-B. In addition to providing access to the lower mediastinum, the oesophageal route has several advantages to the endobronchial approach, including that nodal sampling is easier owing to the absence of cartilage rings and cough. Our findings are comparable with the outcomes of the ASTER trial, in which both EBUS and conventional EUS were used for mediastinal nodal staging [26]. The authors reported a sensitivity of $85 \%$ for mediastinal nodal staging, similar to our $82 \%$. 
In the SCORE study, a systematic and combined approach revealed clinically relevant staging information in one out of 10 patients compared to a targeted EBUS approach. This is relevant because the number of metastatic lymph nodes appears to be a better prognostic determinant than the currently used anatomical location-based $\mathrm{N}$ classification (N0, N1, N2 or N3) in lung cancer patients [27, 28]. A more accurate assessment of nodal status by a systematic EBUS combined with EUS-B is relevant for delineating radiation therapy, which is normally done by PET-CT-based selective lymph node irradiation [29]. The current study was adequately powered and was performed in a large and well-defined study population; the target lesion(s) based on PET-CT imaging was determined prior to endoscopy, and endosonography procedures were performed in a structured and well-documented three-step approach. Patients were included from both general and university hospitals. To avoid the risk of overestimating the accuracy of the endosonography procedures, a conservative analysis was chosen in which only patients with a pathologically proven nodal metastasis were considered true positive. In clinical practice, the diagnosis of $\mathrm{N} 2 / \mathrm{N} 3$ disease is sometimes based only on the combination of PET-CT and endosonography characteristics when nodal aspiration cannot be performed safely (e.g. stations 5 or 6). In this study, EBUS and/or EUS-B detected N2/N3 disease in 90 patients. In 84 patients this was based on tissue proof of nodal metastases. In the other six patients, the diagnosis of malignant involvement was based only on PET-CT and endosonography characteristics (round shape, enlarged, sharp margins and hypo-echogenic), without tissue proof. Pathological proof of N2 disease (all on station 5 and 6) was not obtained by EBUS and/or EUS-B owing to the interposition of vascular structures. For calculation of sensitivity, NPV and NLR, the results of these six patients were considered false negative. If these specific six cases were considered true positives, the sensitivity of targeted EBUS versus systematic EBUS versus combined EBUS+ EUS-B would be $79 \%$ versus $83 \%$ versus $87 \%$, respectively. The NPV would be $85 \%$ versus $87 \%$ versus $90 \%$, respectively.

A limitation of our study was that 36 out of 139 lung cancer patients (26\%) without evidence of mediastinal nodal metastases after endosonography did not undergo surgical nodal verification. This potentially overestimates the sensitivity and NPV of endosonographic procedures. However, clinical and radiological follow-up was available in almost all patients. Using a single EBUS scope for a combined endosonographic staging procedure instead of the using both an EBUS and a separate conventional EUS scope is most likely not a limiting factor, because it has been described previously that both staging approaches are equally effective and safe for mediastinal staging [23, 30].

In conclusion, among patients with (suspected) NSCLC, a systematic staging strategy combining EBUS and EUS-B resulted in increased sensitivity for detecting mediastinal nodal metastases compared with targeted EBUS alone. Moreover, this combined approach resulted in meaningful clinical staging information in $10 \%$ of the included patients. This single EBUS scope and operator staging strategy might prove to be the optimal way to assess locally advanced nodal disease in lung cancer patients. Future studies should confirm current outcomes and investigate the impact on patient management and outcomes.

Conflict of interest: L.M.M. Crombag has nothing to disclose. C. Dooms has nothing to disclose. J.A. Stigt has nothing to disclose. K.G. Tournoy has nothing to disclose. O.C.J. Schuurbiers has nothing to disclose. M.K. Ninaber has nothing to disclose. W.A. Buikhuisen has nothing to disclose. S.M.S. Hashemi has nothing to disclose. P.I. Bonta has nothing to disclose. D.A. Korevaar has nothing to disclose. J.T. Annema has received material and financial support to organise EBUS/EUS courses from Pentax, Hitachi and Cook.

Support statement: This study was funded by Academisch Medisch Centrum. Funding information for this article has been deposited with the Crossref Funder Registry.

\section{References}

1 Howington JA, Blum MG, Chang AC, et al. Treatment of stage I and II non-small cell lung cancer: diagnosis and management of lung cancer, 3rd ed: American College of Chest Physicians evidence-based clinical practice guidelines. Chest 2013; 143: Suppl. 5, e278S-e313S.

2 Ramnath N, Dilling TJ, Harris LJ, et al. Treatment of stage III non-small cell lung cancer: diagnosis and management of lung cancer, 3rd ed: American College of Chest Physicians evidence-based clinical practice guidelines. Chest 2013; 143; Suppl. 5, e314S-e340S.

3 Vansteenkiste J, De RD, Eberhardt WE, et al. Early and locally advanced non-small-cell lung cancer (NSCLC): ESMO clinical practice guidelines for diagnosis, treatment and follow-up. Ann Oncol 2013; 24: Suppl. 6, vi89-98.

4 Schmidt-Hansen M, Baldwin DR, Hasler E, et al. PET-CT for assessing mediastinal lymph node involvement in patients with suspected resectable non-small cell lung cancer. Cochrane Database Syst Rev 2014: CD009519.

5 Silvestri GA, Gonzalez AV, Jantz MA, et al. Methods for staging non-small cell lung cancer: diagnosis and management of lung cancer, 3rd ed: American College of Chest Physicians evidence-based clinical practice guidelines. Chest 2013; 143: Suppl. 5, e211S-e250S.

6 De Leyn P, Dooms C, Kuzdzal J, et al. Revised ESTS guidelines for preoperative mediastinal lymph node staging for non-small-cell lung cancer. Eur J Cardiothorac Surg 2014; 45: 787-798.

7 Vilmann P, Clementsen PF, Colella S, et al. Combined endobronchial and oesophageal endosonography for the diagnosis and staging of lung cancer. European Society of Gastrointestinal Endoscopy (ESGE) guideline, in 
cooperation with the European Respiratory Society (ERS) and the European Society of Thoracic Surgeons (ESTS). Eur Respir J 2015; 46: 40-60.

8 Kinsey CM, Arenberg DA. Endobronchial ultrasound-guided transbronchial needle aspiration for non-small cell lung cancer staging. Am J Respir Crit Care Med 2014; 189: 640-649.

9 Miller RJ, Mudambi L, Vial MR, et al. Evaluation of appropriate mediastinal staging among endobronchial ultrasound bronchoscopists. Ann Am Thorac Soc 2017; 14: 1162-1168.

10 Tournoy KG, Annema JT, Krasnik M, et al. Endoscopic and endobronchial ultrasonography according to the proposed lymph node map definition in the seventh edition of the tumor, node, metastasis classification for lung cancer. J Thorac Oncol 2009; 4: 1576-1584.

11 Liberman M, Sampalis J, Duranceau A, et al. Endosonographic mediastinal lymph node staging of lung cancer. Chest 2014; 146: 389-397.

12 Oki M, Saka H, Ando $M$, et al. Endoscopic ultrasound-guided fine needle aspiration and endobronchial ultrasound-guided transbronchial needle aspiration: are two better than one in mediastinal staging of non-small cell lung cancer? J Thorac Cardiovasc Surg 2014; 148: 1169-1177.

13 Szlubowski A, Zielinski M, Soja J, et al. A combined approach of endobronchial and endoscopic ultrasoundguided needle aspiration in the radiologically normal mediastinum in non-small-cell lung cancer staging - a prospective trial. Eur J Cardiothorac Surg 2010; 37: 1175-1179.

14 Wallace MB, Pascual JM, Raimondo M, et al. Minimally invasive endoscopic staging of suspected lung cancer. JAMA 2008; 299: 540-546.

15 Herth FJ, Krasnik M, Kahn N, et al. Combined endoscopic-endobronchial ultrasound-guided fine-needle aspiration of mediastinal lymph nodes through a single bronchoscope in 150 patients with suspected lung cancer. Chest 2010; 138: 790-794.

16 Hwangbo B, Lee GK, Lee HS, et al. Transbronchial and transesophageal fine-needle aspiration using an ultrasound bronchoscope in mediastinal staging of potentially operable lung cancer. Chest 2010; 138: 795-802.

17 Kang HJ, Hwangbo B, Lee GK, et al. EBUS-centred versus EUS-centred mediastinal staging in lung cancer: a randomised controlled trial. Thorax 2014; 69: 261-268.

18 Goldstraw P, Crowley J, Chansky K, et al. The IASLC Lung Cancer Staging Project: proposals for the revision of the TNM stage groupings in the forthcoming (seventh) edition of the TNM classification of malignant tumours. J Thorac Oncol 2007; 2: 706-714.

19 Konge L, Annema J, Clementsen P, et al. Using virtual-reality simulation to assess performance in endobronchial ultrasound. Respiration 2013; 86: 59-65.

20 Konge L, Vilmann P, Clementsen $\mathrm{P}$, et al. Reliable and valid assessment of competence in endoscopic ultrasonography and fine-needle aspiration for mediastinal staging of non-small cell lung cancer. Endoscopy 2012; 44: 928-933.

21 Annema JT, Versteegh MI, Veselic M, et al. Endoscopic ultrasound added to mediastinoscopy for preoperative staging of patients with lung cancer. JAMA 2005; 294: 931-936.

22 Um SW, Kim HK, Jung SH, et al. Endobronchial ultrasound versus mediastinoscopy for mediastinal nodal staging of non-small-cell lung cancer. J Thorac Oncol 2015; 10: 331-337.

23 Korevaar DA, Crombag LM, Cohen JF, et al. Added value of combined endobronchial and oesophageal endosonography for mediastinal nodal staging in lung cancer: a systematic review and meta-analysis. Lancet Respir Med 2016; 4: 960-968.

24 Block MI. Endobronchial ultrasound for lung cancer staging: how many stations should be sampled? Ann Thorac Surg 2010; 89: 1582-1587.

25 Sanz-Santos J, Serra P, Torky M, et al. Systematic compared with targeted staging with endobronchial ultrasound in patients with lung cancer. Ann Thorac Surg 2018; 106: 398-403.

26 Annema JT, van Meerbeeck JP, Rintoul RC, et al. Mediastinoscopy vs endosonography for mediastinal nodal staging of lung cancer: a randomized trial. JAMA 2010; 304: 2245-2252.

27 Saji H, Tsuboi M, Shimada Y, et al. A proposal for combination of total number and anatomical location of involved lymph nodes for nodal classification in non-small cell lung cancer. Chest 2013; 143: 1618-1625.

28 Wei S, Asamura H, Kawachi R, et al. Which is the better prognostic factor for resected non-small cell lung cancer: the number of metastatic lymph nodes or the currently used nodal stage classification? J Thorac Oncol 2011; 6: 310-318.

29 De Ruysscher D, Wanders S, van Haren E, et al. Selective mediastinal node irradiation based on FDG-PET scan data in patients with non-small-cell lung cancer: a prospective clinical study. Int J Radiat Oncol Biol Phys 2005; 62: 988-994.

30 Szlubowski A, Soja J, Kocon P, et al. A comparison of the combined ultrasound of the mediastinum by use of a single ultrasound bronchoscope versus ultrasound bronchoscope plus ultrasound gastroscope in lung cancer staging: a prospective trial. Interact Cardiovasc Thorac Surg 2012; 15: 442-446. 